\title{
STUDY OF A COMPLETE SAMPLE OF H $\alpha$ EMISSION-LINE GALAXIES FROM THE UCM SURVEY
}

\author{
J. GALLEGO, J. ZAMORANO, M. REGO, A.G. VITORES AND \\ O. ALONSO \\ Dpto. de Astrofísica, Universidad Complutense Madrid, Spain
}

The Universidad Complutense de Madrid survey is a long-term project with the aim of finding and analyzing star forming galaxies using the $\mathrm{H} \alpha$ line as the tracer for star formation processes. In order to obtain a representative and complete sample of the population detected, spectroscopic observations were carried out for the full sample of $\mathrm{H} \alpha$ emission-line galaxy (ELG) candidates of the UCM lists 1 and 2. The ELGs types most commonly found (47\%) are intermediate to low-luminosity objects with a very intense star-formation region which dominates the optical energy output of the galaxy. This kind of ELGs is similar to the galaxy population detected in the blue objective-prism surveys. And what is more important, a second population $(43 \%)$ of star-forming galaxies with low ionization or high extinction properties has been found. This ELGs group is detected neither in the blue (University of Michigan survey, Case survey) nor in other surveys (Kiso, IRAS, Markarian) using other selection techniques.

The position of the different natural groups of ELGs in the diagnostic diagrams suggests that they differ from one another in terms of metal abundance of their ionized gas, the ionization parameter and the relative importance of the starburst process in the galaxy, confirming a previous similar result found for the Michigan survey. Also a trend for lower metallicities at lower luminosities is present. No galaxy with metallicity lower than I $\mathrm{Zw} 18$ has been found.

A study of the luminosity and spatial distribution of the UCM galaxies is presented. The two-point correlation function shows that UCM ELGs follow at great scale the distribution of the catalogue galaxies, although being less clustered in general. Using the UCM ELGs as SFR tracers a luminosity function for the SFR at the local Universe is computed. This result has important implications for our understanding of the origin of the star formation phenomena as well as the evolution of the galaxies. 